Ann. Génét. Sél. anim., I975, 7 (4), 387-408.

\title{
ANALYSE STATISTIQUE ET GÉNETTIQUE DES GAINS DES PUR SANG ANGLAIS DE TROIS ANS DANS LES COURSES PLATES FRANÇAISES
}

\author{
B. LANGLOIS \\ avec la collaboration technique de Dominique Poirec, \\ D. TAstu et J. Rose \\ Département de Génétique animale, \\ Centre national de Recherches zootechniques, I. N. R. A., \\ 78350 Jouy en Josas
}

\section{RÉSUMÉ}

Les gains des Pur sang anglais de 3 ans dans les courses plates françaises de 197 I à 1973 ainsi que ceux de leurs parents au même âge ont été analysés de façon à fournir différentes estimations de l'héritabilité de ce critère.

Pour disposer de variables susceptibles d'être soumises aux calculs statistiques classiques, une transformation logarithmique a été appliquée aux gains de chaque cheval et à son gain moyen par départ. Pour les parents une actualisation à l'année 1973 selon le mode multiplicatif habituel a été réalisée au préalable.

L'analyse de la variance sur les variables transformées portant sur les 5235 descendants gagnants a permis de révéler l'absence d'un effet significatif de l'année et un effet hautement significatif du sexe en faveur des mâles. Par ailleurs l'absence d'interactions entre ces deux facteurs a autorisé la correction des données concernant ces chevaux.

La corrélation phénotypique entre conjoints a été estimée respectivement à $0,23,0,18$, $0,25,0,35$ pour le gain, le gain moyen par départ, le Log du gain et le Log du gain moyen par départ.

La composante étalon de la variance des poulinières conjointes a été estimée à o, 17 pour le $\log$ du gain et à 0,26 pour le Log du gain moyen par départ par une analyse portant sur 3777 juments et 34 I mâles.

Par une autre analyse de variance conduite sur trois échantillons sélectionnés sur un nombre minimum de gagnants par pères de 2,4 et 8 concernant respectivement 5022,4639 et 3777 produits issus de 507,343 et 220 étalons, nous avons pu estimer que la composante paternelle de la variance variait de 0,13 à 0,15 pour le Log du gain et de 0,21 à 0,24 pour le Log du gain moyen par départ.

Les coefficients de régression père-descendant (3 $84 \mathrm{I}$ couples), mère-descendant ( 2728 couples) et parent moyen-descendant ( 2244 couples) ont ensuite été estimés. Des corrections pour les effets de l'homogamie inspirées de la méthode de Fisher (I9I8) ont alors été appliquées aux résultats précédents. Elles ont conduit à des estimations de l'héritabilité très concordantes : les valeurs trouvées toujours inférieures à 0,10 pour les variables non transformées ont été de l'ordre de $0,3 \circ$ pour le Log du gain et de l'ordre de o,40 pour le Log du gain moyen par départ. 


\section{INTRODUCTION}

Depuis des siècles, la sélection des chevaux pour la course passionne les éleveurs et de la pratique de cet art sont nés de nombreux systèmes empiriques d'amélioration génétique. Ainsi la répartition des animaux en lignées plus ou moins nobles remontant jusqu'aux origines, différentes formules de dosage des ancêtres illustres dans les pedigree et diverses utilisations de la consanguinité ou du "croisement " se sont tour à tour disputées la faveur du public. Ces démarches, en grande partie sous l'influence de facteurs émotionnels, n'apparaissent que très partiellement justifiées. En effet, procédant généralement des grands gagnants vers l'ensemble des chevaux soit du particulier au général, elles heurtent bien souvent la logique.

A l'opposé les approches scientifiques du problème, souvent limitées, sont restées du domaine de la théorie et ont rarement gagné l'attention des professionnels. Ce fait étonne d'autant plus que le souci génétique présidant à cet élevage est manifeste et que l'on connaît le succès de ces méthodes chez les autres espèces domestiques. Sans doute faut-il chercher les raisons de cette désaffection dans la consécration de longs usages et dans la situation très particulière de cet animal dans l'agriculture. Toutefois grâce à l'organisation des courses et à l'importance économique qu'elles ont peu à peu acquise, un dispositif inégalé de contrôle des origines et des performances de l'ensemble d'une population s'est progressivement mis en place pour le Pur sang anglais. Il a conduit ses éleveurs qui disposent ainsi de beaucoup d'informations à un choix raisonné des accouplements. Ce phénomène entraînant très probablement des écarts sensibles aux conditions de la panmixie, cela nous conduira par la suite à envisager les problèmes de génétique quantitative sous un angle peu habituel.

Les critères disponibles pour évaluer l'aptitude à la course des chevaux peuvent se regrouper sous les trois rubriques suivantes :

- les temps chronométrés ;

- les valeurs cotées (handicap, time-form, performance-rates...) ;

- les gains.

C'est ce troisième groupe d'estimateurs qui a retenu notre attention pour cette étude. En effet communément employé par les éleveurs français il a encore été relativement peu étudié (PIRRI et STEELE, I95I ; WATANABE et al., I966 ; FoYE et al., 1973).

De plus, les performances des chevaux de 3 ans paraissant de l'avis général et d'après les données de la bibliographie (DUSEK, I966) comme les plus représentatives du potentiel des animaux, nous nous sommes limités à l'analyse génétique du gain et du gain moyen par départ à 3 ans.

Nous nous proposons par la corrélation phénotypique entre conjoints d'estimer l'importance effective de l'homogamie dans la population française de pur sang. Ceci nous permettra par la suite de fournir une série d'estimations de 1'héritabilité de l'aptitude à la course plate en tenant compte de cette particularité. 


\section{I. - MATÉRIEL ET MÉTHODES}

\section{A. - Constitution des fichiers de travail}

I. Les performances des chevaux de 3 ans de 1971 à 1973.

Le relevé des gains de 7577 Pur sang anglais de 3 ans dans les courses plates françaises de I97r à I973 nous a été fourni par la Société d'encouragement à l'élevage des races de chevaux en France. Les variables retenues pour représenter l'aptitude à la course de chaque cheval ont été le gain annuel et le gain moyen annuel par départ.

Par ailleurs $2.34^{2}$ chevaux (30,9 p. I00) n'ayant rien gagné, des individus de potentiel très différent se trouvaient réunis sous la même valeur nulle. Il ne nous a donc pas été possible de les prendre en considération dans cette étude.

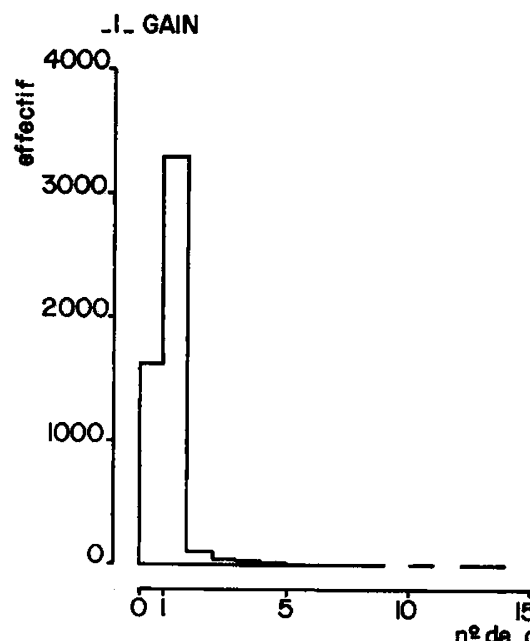

\section{GAIN PAR DEPART}
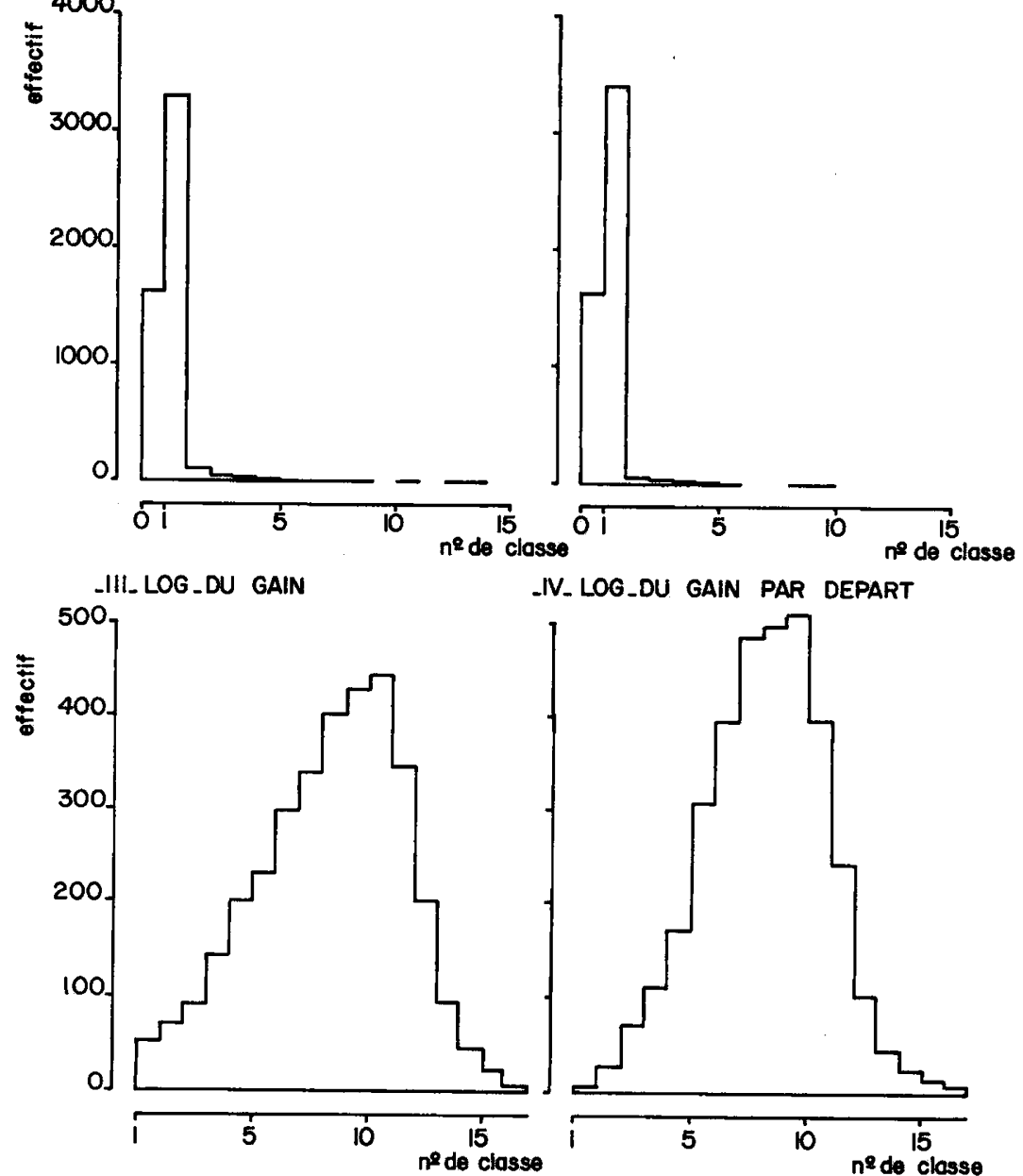

-IV. LOG_DU GAIN PAR DEPART

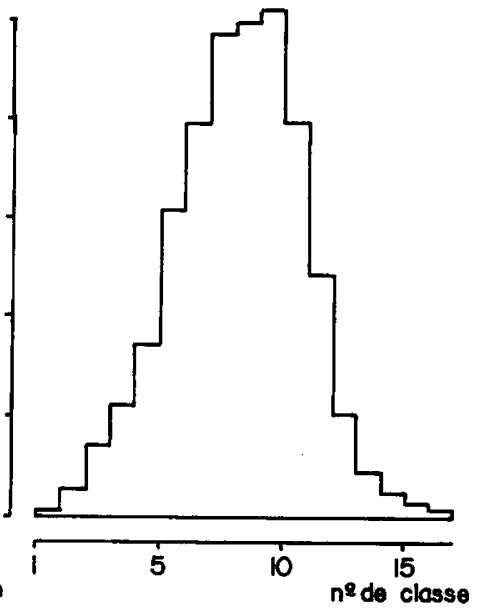

FIG. I. - Distribution de fréquence des variables concernant les chevaux de 3 ans dans les courses plates francaises de 1971 à 1972 
L'examen des distributions de fréquence des deux variables (fig. I), réalisées dans un travail préliminaire sur les 3428 chevaux gagnants en r971 et 1972, a révélé d'emblée leur extrême dissymétrie. Leur transformation a donc dû être envisagée poi:r les soumettre aux analyses statistiques classiques.

Ainsi les fonctions racine carrée, racine cubique, racine quatrième et Logarithme naturel leur ont été appliquées. C'est cette dernière, comme ce fut le cas pour les Concours de Sauts d'Obstacles (LANgloIs, I975), qui a donné les meilleurs résultats (fig. I).

La distribution du Logarithme naturel du gain annuel montre cependant une dissymétrie encore marquée en faveur des gains modestes.

En revanche la distribution du gain moyen par départ apparaît tout à fait satisfaisante. Le test de normalité selon SNEDECOR (1957), auquel elle a été soumise a toutefois révélé qu'elle s'écartait significativement de la normalité par une assymétrie en faveur des faibles valeurs de la variable et un aplatissement trop important.

Il est possible de voir là un effet des courses à handicap qui favoriseraient les chevaux moyens pour le gain annvel. Cet effet serait en partie compensé pour la variable gain moyen par départ, ces " chevaux de handicap " sortant plus que les autres. Cette interprétation qui n'a cependant qu'une valeur d'hypothèse, permet d'expliquer l'échec partiel de la transformation logarithmique, les gains des chevaux n'étant plus distribués en fonction exponentielle de leur valeur.

Ces transformations, malgré la très large amputation des échantillons qu'elles supposent et sur laquelle nous reviendrons dans la discussion, ont néanmoins l'avantage de fournir des critères sur lesquels il sera possible d'estimer par la suite l'effet du sexe, de l'année de la performance et celui du père par les méthodes d'analyse de variance. De plus, réduisant les disproportions considérables des gains, elles permettent vraisemblablement d'obtenir des estimateurs plus convenables du potentiel des chevaux.

\section{Les performances parentales.}

Les 7577 chevaux Pur sang anglais précédents, dont 5235 "étaient dans l'argent ", ont servi de base à la constitution des fichiers de performances de leurs parents.

Les gains et le nombre de courses à 3 ans de 5466 poulinières et 757 étalons ont donc été recherchés manuellement dans les calendriers des courses passées et certains ouvrages édités par l'U.N.I.C. (') (mères de gagnants, répertoire des étalons de pur sang) de façon à disposer des renseignements utiles.


FIG. 2. - Évolution annuelle du nombre

$$
\begin{aligned}
& \text { - de concurrents } \\
& \text { - de partants } \\
& \text { - de départs/cheval -......... }
\end{aligned}
$$

(i) U.N.I.C. : Union Nationale Interprofessionnelle du Cheval. 
Les sommes gagnées en France par ces chevaux (lorsque cela était le cas) ont été ensuite actualisées à l'année 1973 de façon à les rendre comparables. La méthode utilisée, couramment employée, a consisté à multiplier le gain obtenu par un cheval durant l'année $x$ par le rapport de la dotation moyenne par cheval de 1973 sur celle de l'année $x$. Ce principe multiplicatif de correction a été également appliqué au gain moyen par départ, le coefficient utilisé étant alors le rapport des dotations par partant (1). La variation annuelle de ces coefficients est rapportée figure 4. Les figures 2 et 3 qui retracent en parallèle l'évolution générale des courses plates de I94I à I973, nous permettent de mieux apprécier la validité de ces facteurs d'actualisation qui apparaissent discutables mais qui sont aussi les seuls dont nous pouvons disposer aisément.

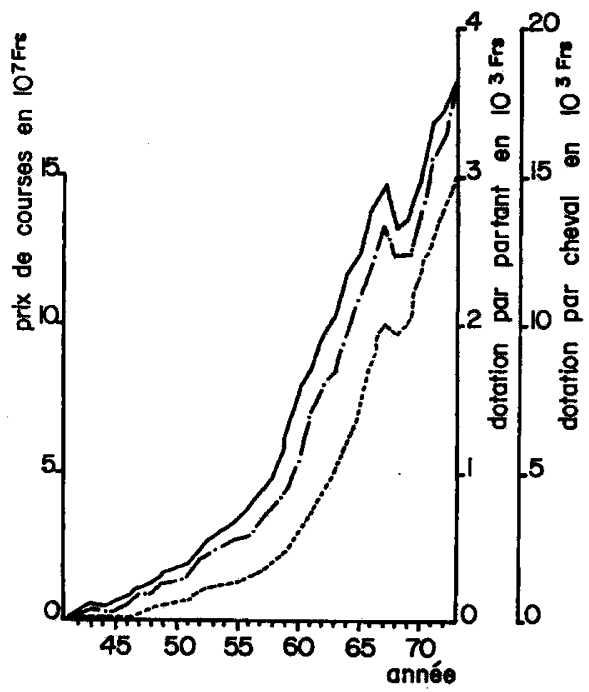

FIG. 3. - Évolution annuelle de la dotation

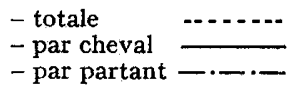

En ce qui concerne le nombre de concurrents tout d'abord, la figure 2 nous montre l'évolution en deux temps de la population des chevaux de course qui, très vite remise des conséquences de la dernière guerre, a atteint vers r950 un premier plateau où elle s'est maintenue jusqu'à r96o, date à laquelle une nouvelle phase de très forte expansion a débuté et s'est perpétuée jusqu'à nos jours. On peut cependant noter un léger ralentissement de cette expansion depuis I968.

L'évolution du nombre de partants s'est déroulée d'une façon tout à fait comparable avec cependant une légère diminution du nombre moyen de départ par cheval de 1948 à 1973 .

Les dotations moyennes par cheval ou par partant qui suivent étroitement la dotation annuelle (fig. 3), se sont en revanche accrues régulièrement sauf en I968 et r 969 où il y a eu diminution.

D'une manière générale on peut dire que de I94I à I973 les effectifs de concurrents ou de partants ont pratiquement quadruplé et les prix de courses se sont trouvé multipliés par 300 .

La courbe des coefficients correctifs (fig. 4) a été tracée en même temps que la distribution des poulinières et étalons concernés de façon à apprécier leur impact sur notre échantillon. Car en effet, si de 1955 à 1970 ces coefficients peuvent être jugés satisfaisants, on peut se demander s'il en est de même de I94I à 1955 étant donné leurs très fortes valeurs et l'éloignement dans le temps des performances considérées. Cependant, comme nous le montre la figure 4 , le faible pourcentage des chevaux concernés laisse supposer que les éventuels incertitudes ou biais qui en résulteraient n'ont sans doute que peu d'influence sur notre échantillon.

(1) I partant $=$ I départ de cheval (un cheval qui prend part à 3 courses correspond à 3 partants). 
Nous avons ainsi pu appliquer la transformation logarithmique à ces gains actualisés afin de disposer sur notre " fichier étalons " et notre "fichier poulinières " des mêmes critères d'estimation de la valeur des chevaux que dans le cas de leurs descendants, soit :

- le gain à 3 ans,

- le gain moyen par départ à 3 ans,

- le Log naturel du gain à 3 ans,

- le Log naturel du gain moyen par départ à 3 ans.

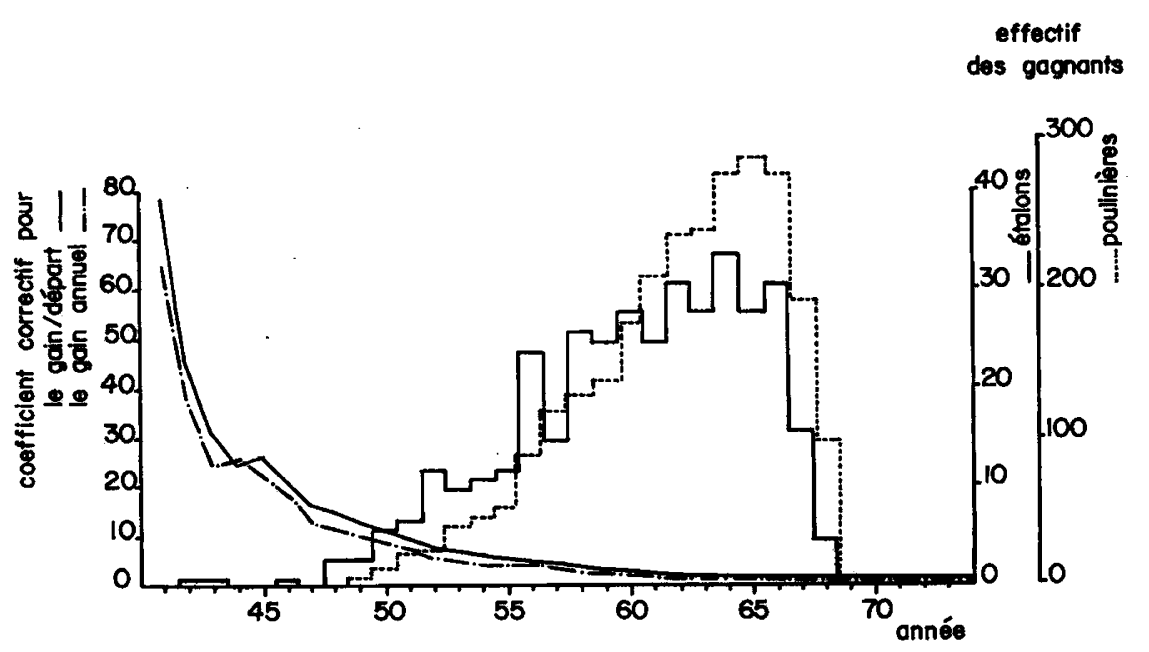

FIG. 4. - Cofficients correctifs des gains pour l'année et effectifs des poulinières et étalons concernés

Pour les produits, la correction des données sera faite pour l'année et le sexe, selon les résultats de l'analyse de la variance sur les 2 variables transformées. En revanche, pour des raisons de commodité dans le traitement des fichiers, les gains bruts ont été laissés tels quels étant donné les facteurs correctifs applicables qui sont très voisins de $x$.

A l'issue de ces différentes opérations, nous avons pu disposer pour les 4 variables précitées des performances de 384 étalons, de 2708 mères et de 5235 produits, ayant gagné en France. Il nous était donc possible d'envisager différents modes d'estimation de l'héritabilité et c'est le détail de ces méthodes d'estimation que nous proposons d'exposer maintenant.

\section{B. - Méthodes}

Les méthodes que nous nous proposons d'employer pour estimer l'héritabilité sont d'une part celles utilisant les régressions du phénotype des descendants sur celui des parents et d'autre part celle utilisant la composante paternelle de la variance phénotypique des descendants.

\section{Méthodes de régression.}

- En panmixie nous savons que l'héritabilité peut être obtenue en doublant le coefficient de régression linéaire du phénotype des descendants sur celui du père $(b d / p)$ ou de la mère $(b d / m)$ soit :

$$
\begin{aligned}
& h^{2}=2 b d / p \\
& h^{2}=2 b d / m
\end{aligned}
$$

En revanche, quand il existe une homogamie phénotypique dans la population, FisHER (I9I8) a montré que ces estimations devaient être corrigées pour tenir compte de ce phénomène.

En effet, dans le cas de croisements assortis, si le père dévie d'une unité par rapport à la moyenne de la population, la mère en raison de la corrélation entre conjoints déviera de 
$b m / p$, coefficient de régression du phénotype de la mère sur celui du père. La déviation moyenne des deux parents sera alors $(\mathrm{I}+b \mathrm{~m} / \mathrm{p}) \frac{\mathbf{I}}{2}$ et la déviation attendue pour les descendants sera la partie génétique de celle-ci, soit $h^{2}$ fois la déviation parentale moyenne. Pour cette raison $b d / p$ s'écrira à l'équilibre des croisements assortis :

$$
b d / p=\frac{\mathrm{I}}{2} h^{2}(\mathrm{I}+b m / p)
$$

d'où :

$$
h^{2}=\frac{2 b d / p}{I+b m / p}
$$

Le même raisonnement pour la régression du phénotype des descendants sur celui des mères reste valable et conduit à l'équation suivante :

$$
h^{2}=\frac{2 b d / m}{\mathrm{I}+b p / m}
$$

où $b \mathrm{p} / \mathrm{m}$ est le coefficient de régression du phénotype des pères sur celui des mères.

Il faut noter de surcroît que ces estimations sont celles de l'héritabilité réalisée à l'équilibre dans le cas d'une population homogame et qu'elle diffère de celle qui résulterait de la panmixie pour cette même population. En effet Fisher (1918) a mis en évidence que si le nombre de gènes impliqués dans la réalisation du caractère est suffisamment grand, l'homogamie augmente la variance génétique additive de la population et modifie peu les variances imputables à la dominance et au milieu. Elle contribue donc à augmenter l'héritabilité.

- Une autre méthode classique pour estimer l'héritabilité est l'utilisation du coefficient de régression du phénotype du descendant sur la moyenne de ceux de ses deux parents $(b d / \bar{p})$ et dans ces conditions :

$$
h^{2}=b d / \bar{p}
$$

REEVE (I955, I96I) a montré que cette méthode était très robuste dans le cas des croisements assortis et que les estimations ne s'en trouvaient que fort peu modifiées. Ce résultat peut d'ailleurs être redémontré sans difficulté à l'aide des équations (3) et (4) selon Fisher (1918).

- La dernière possibilité est l'utilisation de l'équation de régression multiple suivante prédisant la valeur phénotypique d'un descendant à partir de celles de ses parents.

$$
\mathrm{D}=b d / p \cdot m \mathrm{P}+b d / m \cdot p \mathrm{M}+b .
$$

D est la valeur phénotypique du descendant;

$P$ est la valeur phénotypique du père ;

$M$ est la valeur phénotypique de la mère.

$$
b d / p \cdot m=\frac{\operatorname{Var}(\mathrm{M}) \operatorname{cov}(\mathrm{P}, \mathrm{D})-\operatorname{cov}(\mathrm{P}, \mathrm{M}) \operatorname{cov}(\mathrm{M}, \mathrm{D})}{\operatorname{Var}(\mathrm{P}) \operatorname{Var}(\mathrm{M}) \operatorname{cov}(\mathrm{P}, \mathrm{M})^{2}}
$$

est le coefficient de régression de la valeur phénotypique des descendants sur celle des pères, à mère constante.

$$
b d / m \cdot p=\frac{\operatorname{Var}(\mathrm{P}) \operatorname{cov}(\mathrm{M}, \mathrm{D})-\operatorname{cov}(\mathrm{P}, \mathrm{M}) \operatorname{cov}(\mathrm{D}, \mathrm{P})}{\operatorname{Var}(\mathrm{P}) \operatorname{Var}(\mathrm{M})-\operatorname{cov}(\mathrm{P}, \mathrm{M})^{2}}
$$

est le coefficient de régression de la valeur phénotypique des descendants sur celle des mères, à père constant,

$b$ est une constante dépendant des moyennes phénotypiques des parents et descendants.

Ces deux coefficients de régression partielle éliminant l'effet de l'homogamie puisqu'ils sont à conjoint constant, fournissent également comme on peut le démontrer en utilisant les équations (3) et (4), une estimation de l'héritabilité par doublement. Nous pouvons donc écrire :

$$
\begin{aligned}
& h^{2}=2 b d / p \cdot m \\
& h^{2}=2 b d / m \cdot p
\end{aligned}
$$


Rappelons que toutes ces estimations supposent d'une part l'absence d'interactions géniques (effets de dominance et d'épistasie) communes à chacun des parents utilisé et au descendant, soit d'une manière générale l'absence de consanguinité dans les accouplements, d'autre part l'absence d'effets de milieu commun sur chacun des parents utilisé et sur le descendant.

Par ailleurs en toute rigueur elles supposent également l'absence de sélection de chacun des parents utilisé mais les biais dus à ce fait sont faibles (RoNNINGEN, 1972).

\section{Méthode utilisant la composante paternelle de la variance.}

L'extension des raisonnements de Fisher (I9I8) dans le cas de la composante paternelle de la variance est exposée en annexe à cet article. Elle nous a conduit dans le cas d'une population homogame à proposer une équation du second degré pour estimer l'héritabilité dont l'expression est la suivante :

$$
\left(2 r \sqrt{\mathrm{K}}+\mathrm{K} t_{m}\right) h^{4}+h^{2}-{ }_{4} \mathbf{R} t_{d}=0
$$

dans laquelle :

$h^{2}$ est l'héritabilité,

$t_{d}$ est la composante paternelle de la variance phénotypique des descendants,

$t_{m}$ est la corrélation intra-classe de père du phénotype des conjointes,

$r$ est la corrélation phénotypique entre conjoints,

$\mathrm{K}$ est le rapport de la variance génétique additive chez les mères sur celle des pères. $K$ peut être approché par les variances phénotypiques correspondantes,

$R$ est le rapport de la variance phénotypique des descendants sur celle des pères.

Remarquons enfin que les hypothèses sous-jacentes à ces estimations sont l'absence de croisements consanguins et d'effets de milieu commun sur les descendants d'un même étalon. La condition classique de pères non sélectionnés peut être abandonnée en partie en raison de la prise en compte du coefficient R. Il faut toutefois pouvoir admettre que l'héritabilité chez les pères sélectionnés est la même que pour l'ensemble de la population. Car en effet c'est le rapport de la variance génétique additive chez les pères sur la variance phénotypique correspondante qui se trouve ainsi mesurée.

\section{II. - RÉSULTATS}

\section{A. - Estimation des effets du sexe et de l'année sur les performances des chevaux de 3 ans de 1971 à 1973}

Les deux variables transformées ont été classées en fonction du sexe (trois niveaux : mâles, femelles et hongres) et de l'année de la performance réalisée (trois niveaux : I97I, I972 et 1973) pour être soumises à une analyse de variance par la méthode des moindres carrés.

La première moitié du tableau I donne les résultats de cette analyse. Il y apparaît une action hautement significative du sexe, l'absence d'effet année et l'absence d'interaction entre les deux facteurs.

Les effets moyens du sexe sur les deux variables mettent en évidence la supériorité des mâles sur les femelles et les hongres qui se révèlent être les moins bons. Il nous a donc paru opportun, sachant que dans les écuries de course seuls les mâles médiocres sont castrés, de refaire une analyse semblable avec seulement deux niveaux pour le sexe (mâles + hongres et femelles). Les résultats en sont fournis dans la deuxième moitié du tableau $\mathrm{I}$ et demeurent comparables aux précédents, l'effet du sexe restant hautement significatif. C'est d'après ces derniers éléments que nous avons pu corriger pour ces effets les performances des chevaux de 3 ans de 197I à 1973 avant de les soumettre aux analyses génétiques. 
TABLEAU I

Résultats des analyses de la variance en fonction de l'année et du sexe sur les performances des chevaux de 3 ans de 1971 à 1973

\begin{tabular}{l|l|l}
\hline 1re analyse (6 niveaux) & $2^{\mathrm{e}}$ analyse (5 niveaux) \\
\hline
\end{tabular}

Tableaux des carrés moyens

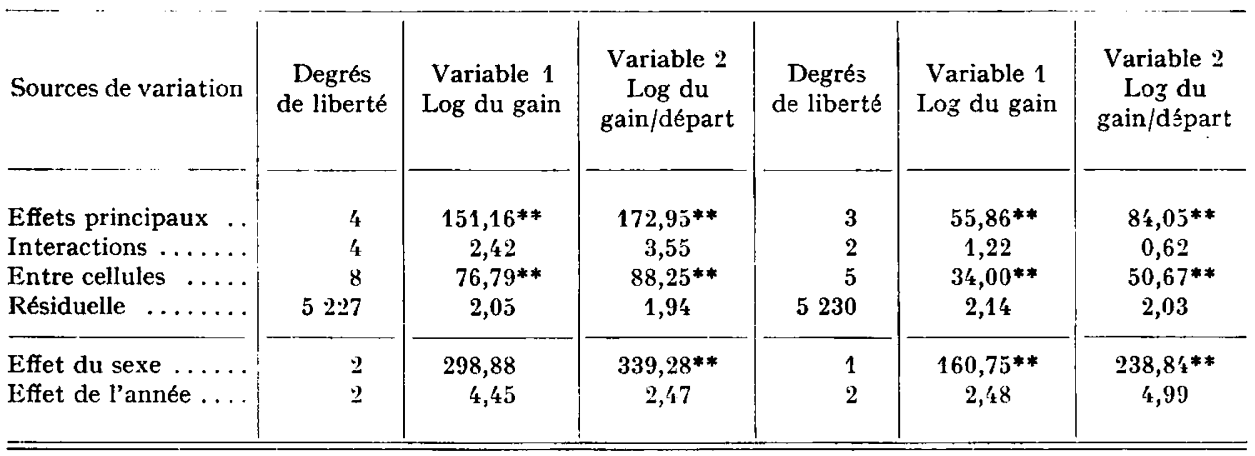

Tableaux des effets moyens des facteurs

\begin{tabular}{|c|c|c|c|c|c|c|}
\hline Effets moyens des & $\begin{array}{c}\text { Effectifs } \\
\text { par niveau }\end{array}$ & $\begin{array}{c}\text { Variable } 1 \\
\text { moy. }=9,53\end{array}$ & $\begin{array}{l}\text { Variable } 2 \\
\text { moy. }=7,71\end{array}$ & $\begin{array}{c}\text { Effectifs } \\
\text { par niveau }\end{array}$ & $\begin{array}{c}\text { Variable } 1 \\
\text { moy. }=9,52\end{array}$ & $\begin{array}{c}\text { Variable } 2 \\
\text { moy. }=7,68\end{array}$ \\
\hline $\begin{array}{l}\text { Mâles } \ldots \ldots \ldots \ldots \\
\text { Hongres } \ldots \ldots \ldots \ldots \\
\text { Femelles } \ldots \ldots \ldots \ldots\end{array}$ & $\begin{array}{l}1605 \\
1127 \\
2504\end{array}$ & $\begin{array}{r}0,50 \\
-0,31 \\
-0,19\end{array}$ & $\begin{array}{r}0,53 \\
-0,29 \\
-0,24\end{array}$ & $\begin{array}{l}2732 \\
2504\end{array}$ & $\begin{array}{r}0,18 \\
-0,18\end{array}$ & $\begin{array}{r}0,21 \\
-0,21\end{array}$ \\
\hline $\begin{array}{l}1971 \ldots \ldots \ldots \\
1972 \ldots \ldots \ldots \ldots \\
1973 \ldots \ldots \ldots \ldots\end{array}$ & $\begin{array}{ll}1651 \\
1777 \\
1808\end{array}$ & $\begin{array}{r}0,05 \\
-0,04 \\
-0,01\end{array}$ & $\begin{array}{r}0,03 \\
-0,0 \prime \\
0,01\end{array}$ & $\begin{array}{ll}1 & 651 \\
1 & 777 \\
1 & 808\end{array}$ & $\begin{array}{r}0,00 \\
-0,0^{\prime} \\
0,0^{\prime}\end{array}$ & $\begin{array}{r}0,02 \\
-0,01 \\
0,03\end{array}$ \\
\hline
\end{tabular}

** Effet significatif au seuil de 1 p. 100.

\section{B. - Estimation de l'héritabilité}

par les régressions simples du phénotype des descendants sur celui des parents

Nous avons vu précédemment comment la constitution des fichiers de performances sur les pères et mères des chevaux ayant eu 3 ans de I97I à I973 nous permettait d'appliquer ces méthodes. Nous avons pu disposer ainsi pour les 4 variables considérées de :

- $384 \mathrm{I}$ couples père-descendant ;

- 2728 couples mère-descendant ;

- 2244 couples parent moyen-descendant. 
B. LANGLOIS




GAINS DES " PUR SANG ANGIAIS "

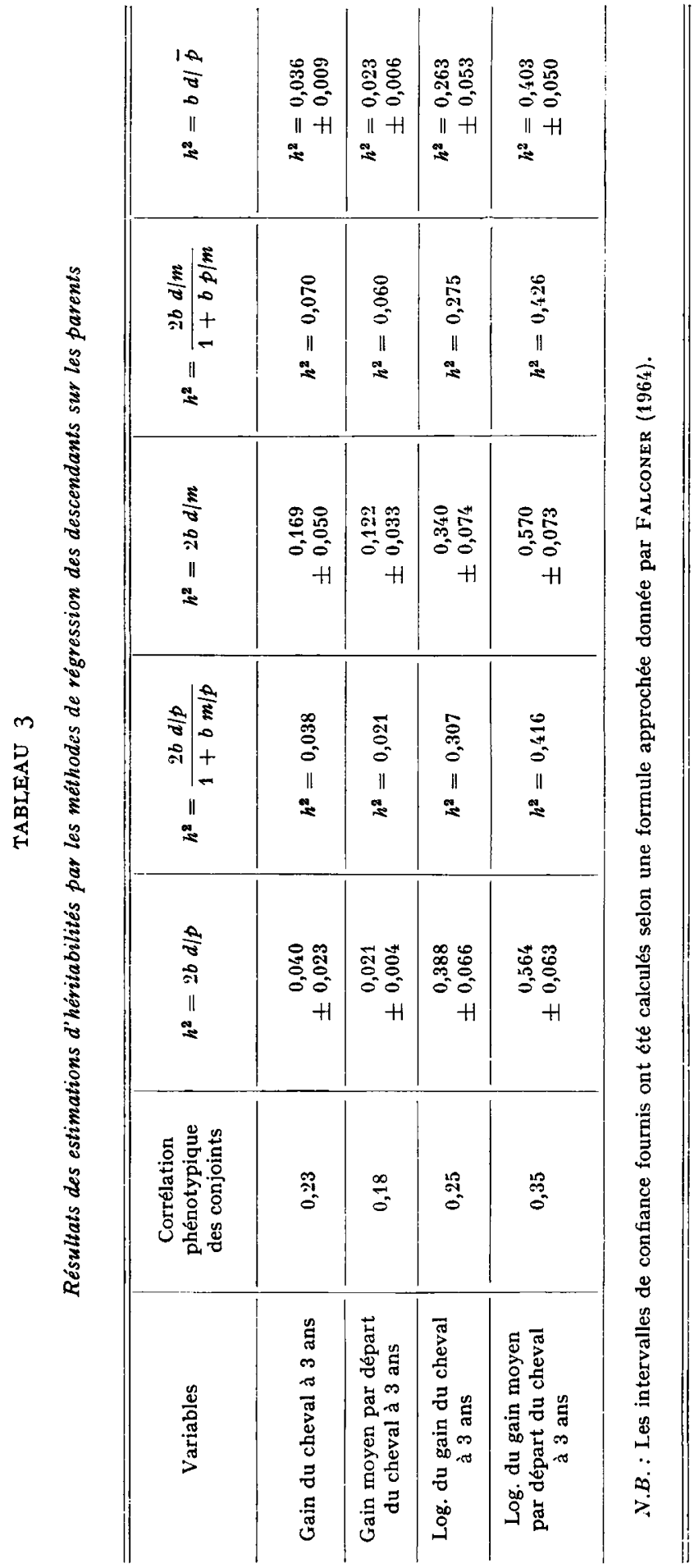


sur lesquels toutes les moyennes variances et covariances utiles ont été calculées (tabl. 2). L'exploitation de ces résultats nous a tout d'abord conduit à mettre en évidence pour les 4 variables des corrélations phénotypiques hautement significatives entre conjoints qui oscillent entre 0,18 et 0,35 (tabl. 3). Par la suite, ces valeurs justifiant l'emploi des corrections exposées précédemment dans le cas d'accouplements assortis, nous avons pu proposer une série d'estimations de l'héritabilité. Le tableau 3 récapitule l'ensemble des valeurs trouvées qui convergent assez bien sur une héritabilité toujours inférieure à 0,10 des variables non transformées et une héritabilité de l'ordre de 0,30 pour le logarithme du gain et de 0,40 pour le logarithme du gain moyen par départ. Nous pouvons également remarquer que la prise en considération de l'homogamie fait considérablement chuter les estimations obtenues par les méthodes classiques, même pour des corrélations entre parents qui n'apparaissent pas a priori très importantes (tabl. 3).

\section{C. - Utilisation de la régression multiple}

Nous ne nous sommes intéressés à cette méthode que dans le cas des deux variables transformées dont les distributions plus proches de la normalité paraissaient mieux convenir.

Connaissant la matrice de variance et covariance des 3 variables suivantes, phénotype du père, $P$, phénotype de la mère, $M$, phénotype du produit, $D$, il nous était donc possible de calculer l'équation de régression multiple prédisant statistiquement la valeur d'un produit à partir de celle de son père et de sa mère.

Les résultats des calculs sur les 2244 séries de données déjà utilisées dans le calcul de la régression parent moyen-descendant, ont fourni les équations suivantes :

$$
\widehat{\mathrm{D}}=0, \mathrm{I5} \mathrm{P}+0, \mathrm{I} 2 \mathrm{M}-0,84
$$

avec :

$$
\mathrm{R}^{2}(\mathrm{D}, \mathrm{P} \cdot \mathrm{M})=0,27
$$

pour la variable logarithme naturel du gain du cheval à 3 ans.

et :

$$
\widehat{\mathrm{D}}=0,20 \mathrm{P}+0,20 \mathrm{M}+3,9 \mathrm{I}
$$

avec :

$$
\mathbf{R}^{2}(\mathrm{D}, \mathbf{P} \cdot \mathrm{M})=0,98
$$

pour la variable Logarithme naturel du gain moyen par départ du Cheval à 3 ans, où $\widehat{D}$ est l'estimation de $\mathrm{D}$ phénotype du descendant, et où $R^{2}(D, P \cdot M)$ est le coefficient de détermination de la régression multiple, soit un indice de précision.

Notons que dans le cas du Log du gain moyen par départ, ce coefficient est très élevé et que la connaissance de la valeur des parents détermine pratiquement celle du descendant. Il serait ainsi possible, à la limite, de prédire à $\mathrm{I} / 3$ de non gagnants près, l'espérance de gain d'un yearling. Notons cependant que ce fort coefficient de détermination tient compte de l'homogamie phénotypique importante dans la population et que dans tel ou tel cas particulier, il pourrait être pris en défaut! De plus des effets systématiques de milieu intervenant pour augmenter la précision de ces estimations ne sont pas à écarter. Des sous-populations isolées suivant l'échelle des 
gains des animaux seraient peut-être à considérer! En effet la prise en compte de l'hérédité et de l'homogamie n'apparaît pas a priori suffisante pour expliquer un tel coefficient de détermination.

Les estimations de l'héritabilité à partir des coefficients de régression partielle sont 0,30 et 0,24 pour le Logarithme du gain et 0,40 et 0,40 pour le Logarithme du gain moyen par départ. Elles concordent parfaitement avec les estimations précédentes.

\section{D. - Utilisation de la composante paternelle de la variance}

r. Estimation de $t_{d}$ la composante paternelle de la variance phénotypique des descendants.

Un modèle d'analyse hiérarchique père-individu selon KEMPTHORNE (I957), a été appliqué aux variables transformées corrigées pour les effets du sexe ( 2 niveaux) et de l'année de la performance (3 niveaux).

Trois échantillons de la population de départ sélectionnés sur un nombre minimum de descendants gagnants par père respectivement de 8,4 et 2 ont été retenus pour les calculs. Les résultats sont rapportés au tableau 4.

\section{TABLEAU 4}

Résultats de l'analyse hiérarchique

\begin{tabular}{|c|c|c|c|}
\hline Échantillon & 1 & 2 & 3 \\
\hline $\begin{array}{l}\text { Critère de sélection } \\
\text { de l'échantillon }\end{array}$ & $\begin{array}{l}\text { Pères ayant au moins } \\
8 \text { descendants } \\
\text { gagnants }\end{array}$ & $\begin{array}{l}\text { Pères ayant au moins } \\
4 \text { descendants } \\
\text { gagnants }\end{array}$ & $\begin{array}{l}\text { Pères ayant au moins } \\
2 \text { descendants } \\
\text { gagnants }\end{array}$ \\
\hline $\begin{array}{l}\text { Nombre de pères } \ldots \ldots \ldots \ldots \ldots \ldots \\
\text { Nombre de descendants } \ldots \ldots \ldots\end{array}$ & $\begin{array}{r}220 \\
3777\end{array}$ & $\begin{array}{r}343 \\
4639\end{array}$ & $\begin{array}{r}507 \\
5022\end{array}$ \\
\hline $\begin{array}{l}\text { Composante paternelle de la va- } \\
\text { riance pour la variable } 1(\mathbf{1}) \ldots \ldots \\
\text { Résiduelle pour la variable } 1 \ldots \ldots\end{array}$ & $\begin{array}{l}0,258^{* *}\left({ }^{3}\right) \\
1,794\end{array}$ & $\begin{array}{l}0,301 * * \\
1,815\end{array}$ & $\begin{array}{l}0,319^{* *} \\
1,804\end{array}$ \\
\hline $\begin{array}{l}\text { Composante paternelle de la va- } \\
\text { riance en p. } 100\left(t_{d}\right) \text { variable } 1 \ldots\end{array}$ & 12,6 & 14,2 & 15,0 \\
\hline $\begin{array}{l}\text { Composante paternelle de la va- } \\
\text { riance pour la variable } 2\left({ }^{2}\right) \ldots \ldots \\
\text { Résiduelle pour la variable } 2 \ldots \ldots\end{array}$ & $\begin{array}{l}0,399 \\
1,504\end{array}$ & $\begin{array}{l}0,456^{* *} \\
1,544\end{array}$ & $\begin{array}{l}0,475 * * \\
1,542\end{array}$ \\
\hline $\begin{array}{l}\text { Composante paternelle de la va- } \\
\text { riance en p. } 100\left(t_{d}\right) \text { variable } 2 \ldots\end{array}$ & 20,9 & 22,8 & 23,5 \\
\hline
\end{tabular}

(1) Variable 1 = Logarithme naturel du gain par cheval.

( $\left.{ }^{2}\right)$ Variable $2=$ Logarithme naturel du gain moyen par départ.

(3) ** : Effet significatif au seuil de 1 p. 100. 
Il apparaît que l'abaissement du nombre minimum de produits par étalon entraîne une légère augmentation de la composante paternelle. Vraisemblablement moins les descendants d'un étalon sont nombreux, plus ils ont de chance de se ressembler.

Nous pensons néanmoins que la valeur $t_{d}=0, \mathrm{I} 4$ peut être retenue pour la variable Logarithme naturel $\mathrm{du}$ gain et la valeur $t_{d}=0,23$ peut l'être pour la variable Logarithme naturel du gain moyen par départ.

2. Estimation de $t_{m}$ composante étalon des valeurs phénotypiques des conjointes.

Comme au paragraphe précédent, les valeurs phénotypiques des mères ont été soumises à une analyse hiérarchique mère intra-étalon selon KEMPTHORNE (I957). Le tableau 5 donne les résultats de cette analyse.

\section{TABLEAU 5}

Résultats de l'analyse hiérarchique mère intra-étalon selon KEMPTHORNE (1957)

\begin{tabular}{l|c|c|c}
\hline $\begin{array}{c}\text { Composantes } \\
\text { de la variance }\end{array}$ & $\begin{array}{c}\text { Degrés } \\
\text { de liberté }\end{array}$ & $\begin{array}{c}\text { Log du gain (1) } \\
\text { du cheval à 3 ans }\end{array}$ & $\begin{array}{c}\text { Log du gain (1) } \\
\text { moyen par départ } \\
\text { du cheval à 3 ans }\end{array}$ \\
\hline $\begin{array}{l}\text { due à l'étalon } \\
\text { résiduelle }\end{array}$ & 341 & $0,386^{* *}$ & $0,555^{* *}$ \\
$\begin{array}{l}\text { due à l'étalon } \\
\text { en p. } 100\left(t_{m}\right)\end{array}$ & $-1,888$ & 1,574 \\
\hline
\end{tabular}

(1) Il s'agit des gains corrigés pour les fluctuations annuelles de la dotation par cheval et par partant.

Les valeurs de $t_{m}$ ainsi trouvées, après sélection des pères accouplés au moins à deux poulinières gagnantes, ont été de 0,17 pour le Log du gain et de 0,26 pour le Log du gain moyen par départ.

Ces valeurs notamment $t_{m}=0,26$ qui exprime pour le Logarithme naturel du gain moyen par départ la répétabilité du phénotype de la jument intra-étalon, paraît tout à fait intéressante. Elle laisse supposer, en effet, que la connaissance d'un nombre relativement faible de juments accouplées à un étalon, pourrait fournir une estimation assez précise du niveau de sa jumenterie. Ce phénomène n'était pas prévisible car les valeurs 0,17 et 0,26 sont supérieures à celles qu'il était permis d'attendre soit $(0,25)^{2}=0,06$ et $(0,35)^{2}=0,12$. L'homogamie chez le pur sang n'est donc pas le seul phénomène à prendre en considération et l'on doit tenir compte de l'homogénéisation importante des " harem " d'étalons qui vient à sa suite.

\section{Estimation de l'héritabilité.}

L'équation de calcul est, rappelons le, la suivante :

$$
\left(2 r \sqrt{\mathrm{K}}+\mathrm{K} t_{m}\right) h^{4}+h^{2}-{ }_{4} \mathrm{R} t_{d}=0
$$


dans laquelle :

- $t_{d}$ : coefficient de corrélation intra-classe de père du phénotype des descendants, est $0, I_{4}$ pour le Log du gain et 0,23 pour le Log du gain moyen par départ;

- $r$ : corrélation phénotypique entre conjoints, est 0,25 pour le Log du gain et 0,35 pour le Log du gain moyen par départ;

- $t_{m}$ : coefficient de corrélation intra-classe d'étalon des valeurs phénotypiques des mères, est 0,17 pour le Log du gain et 0,26 pour le Log du gain moyen par départ ;

- $\mathrm{K}=\operatorname{Var}\left(\mathrm{A}_{m}\right) / \operatorname{Var}\left(\mathrm{A}_{p}\right)$ peut être estimé selon le tableau 2 par le rapport des variances phénotypiques des mères à celle des pères soit I, Io pour le Log du gain moyen par départ;

- $\mathrm{R}$ : rapport de la variance phénotypique des descendants à celle des pères a été également estimé en fonction des données du tableau 2 à I,03 pour le Log du gain et à 0,95 pour le Log du gain moyen par départ.

Il découle de ces valeurs que l'estimation de l'héritabilité par la méthode de la composante paternelle de la variance est de 0,44 pour le Log du gain du cheval à 3 ans et de 0,56 pour le Log du gain moyen par départ du cheval à 3 ans. Ces résultats nettement plus élevés que les précédents laissent supposer une grande influence d'effets de milieu commun sur la descendance des étalons. En effet si nous supposons que les valeurs 0,30 et 0,40 sont de bonnes estimations de 1 'héritabilité nous pouvons selon la formule (3) de l'annexe estimer l'écart entre la composante paternelle de la variance et son estimation en fonction des corrélations génétiques entre conjoints et entre poulinières d'un même étalon. Les différences observées soit 0,05 pour le Log du gain et 0,08 pour le Log du gain moyen par départ sont importantes et laissent supposer que chez ces chevaux de course un tiers environ de la ressemblance entre demi-frères de père n'est pas dû aux covariances des valeurs génétiques.

Pour conclure, nous avons rassemblé au tableau 6 l'ensemble des estimations de l'héritabilité que nous venons d'énumérer. Cela illustre la bonne concordance générale des résultats.

\section{TABLEAU 6}

Récapitulatif des estimations de l'héritabilité

\begin{tabular}{|c|c|c|c|c|c|}
\hline Méthode & Formules & Gains & $\begin{array}{l}\text { Gains par } \\
\text { épreuve }\end{array}$ & Log gain & $\begin{array}{c}\text { Log gain } \\
\text { par } \\
\text { épreuve }\end{array}$ \\
\hline Régressions & $\begin{array}{l}h^{2}=2 b d / p / 1+b m / p \\
h^{2}=2 b d / m / 1+b p / m \\
h^{2}=b d / \bar{p} \\
h^{2}=2 b d / p \cdot m \\
h^{2}=2 b d / m \cdot p\end{array}$ & $\begin{array}{c}0,04 \\
0,07 \\
0,04 \\
- \\
-\end{array}$ & $\begin{array}{l}0,02 \\
0,06 \\
0,02 \\
- \\
-\end{array}$ & $\begin{array}{l}0,31 \\
0,28 \\
0,26 \\
0,30 \\
0,24\end{array}$ & $\begin{array}{l}0,42 \\
0,43 \\
0,40 \\
0,40 \\
0,40\end{array}$ \\
\hline $\begin{array}{c}\text { Composante } \\
\text { paternelle }\end{array}$ & $\left(2 r \sqrt{\mathrm{K}}+\mathrm{K} t_{m}\right) h^{4}+h^{2}-4 \mathrm{R} t_{d}=0$ & - & - & $0,4^{\prime} 4$ & 0,56 \\
\hline
\end{tabular}




\section{III. - DISCUSSION GÉNÉRALE ET CONCLUSIONS}

Les hypothèses sur lesquelles sont fondées nos estimations de l'héritabilité sont nombreuses. Elles peuvent néanmoins être regroupées sous les trois principales rubriques suivantes : 1 'absence de consanguinité, l'absence d'effets de milieu commun et 1'absence de sélection.

Pour le premier point, la corrélation phénotypique entre conjoints $r=0,35$ pour la variable Logarithme naturel du gain moyen par départ d'héritabilité $h^{2}=0,40$ ne laisse entrevoir qu'une corrélation génétique $r_{\mathrm{A}}=h^{2} r$ de $0, \mathrm{I} 4$ ce qui est faible. En effet, la prétendue consanguinité du Pur sang anglais, pratiquée de temps à autre, ne porte dans la majorité des cas que sur des ancêtres communs éloignés. Cela ne peut suffire à introduire dans nos estimations des biais importants du fait de la réalisation d'interactions géniques semblables dans les différents groupes parentaux utilisés. En d'autres termes il nous paraît qu'effectivement les covariances des effets génétiques non additifs peuvent être négligées comme nous l'avons fait entre pères et descendants, mères et descendants et même, bien que ce soit plus discutable, entre descendants d'un même père. Notons enfin que l'existence de telles covariances conduirait, par surestimation des covariances génétiques additives, à des sur évaluations de l'héritabilité.

De la même façon pouvons-nous justifier qu'entre pères et descendants, mères et descendants et descendants d'un même père les effets de milieu commun sont négligeables?

En ce qui concerne les parents et leurs produits, l'intervalle de génération chez le Pur sang anglais étant de l'ordre de Io ans pour les deux voies paternelle et maternelle, il y a en effet peu de chance qu'ils aient bénéficiés de conditions de milieu identiques. Une réserve, cependant, peut être formulée pour les mères et leurs descendants, certains haras assurant le renouvellement de leurs poulinières par leurs propres pouliches. Cependant une covariance de la valeur phénotypique des parents avec l'effet du milieu sur les descendants n'est pas à exclure, les produits de bons " performers " bénéficiant sans doute de soins plus attentifs. Cela conduirait dans l'ensemble à surévaluer l'héritabilité par les méthodes de régression. Il est probable également que la production de certains étalons bénéficie de milieux préférentiels d'élevage et de mise en valeur des produits. L'estimation de l'héritabilité par la composante paternelle de la variance conduirait donc, comme nos résultats semblent d'ailleurs le confimer, à des valeurs trop fortes. En résumé, les effets du milieu dans le cas du Pur sang anglais ne semblent pas se répartir au hasard sur tous les génotypes et cela conduit probablement à surestimer l'importance des effets génétiques.

L'absence de sélection des pères, des mères et de leurs descendants constitue le troisième point à discuter. Deux aspects sont à envisager ici, celui du critère utilisé, seuls les chevaux gagnants sont pris en compte, et celui dû au souci d'amélioration génétique des éleveurs.

Dans le premier cas, la question qui se pose est de savoir si les chevaux gagnants sont représentatifs de l'ensemble de la population ou au contraire s'ils en 
constituent un échantillonnage sélectionné. C'est vers la seconde hypothèse que l'on incline de prime abord et alors toutes les covariances phénotypiques dont nous nous sommes servis dans les calculs précédents, entre parents, entre les conjointes d'un même étalon, entre parents et descendants, entre descendants d'un même père, sont probablement surestimées. Notons cependant que ces différents biais semblent se contrebalancer, au moins partiellement, dans les différentes estimations de 1'héritabilité proposées dans le cadre d'une population homogame.

Remarquons par ailleurs que $2 / 3$ des chevaux partants ne représentent pas un taux de sélection très élevé surtout si l'on considère qu'il n'y a pas véritablement troncature. En effet, tout pur sang " sain et net " est capable de se placer, si l'on s'en donne la peine, dans une petite course. De même, l'importance des effets de milieu de type tout ou rien sur les aptitudes sportives recherchées chez ces animaux explique une grande partie des gains nuls. On est alors tenté d'après ces considérations de revenir à la première hypothèse et de considérer qu'un cheval qui n'a rien gagné est un cheval qui n'a pas pu véritablement s'exprimer.

L'absence de sélection par les éleveurs des étalons et des mères est le second aspect qu'il nous faut maintenant préciser.

Cette hypothèse bien que discutable peut être envisagée pour les juments. En effet l'expansion de la population de pur sang et la faible fertilité des poulinières n'autorise qu'un taux de sélection réduit comme 1'indique le chiffre approximatif de 0,85 obtenu, dans l'hypothèse de normalité, par comparaison de la moyenne des mères à celle de leurs descendants pour la variable Logarithme du gain moyen par départ (tabl. 2).

A l'inverse cette hypothèse ne peut être admise pour les étalons pour lesquels le même taux a été approximativement estimé dans les mêmes conditions à 0,04 . Nous sommes donc amenés à en examiner les conséquences.

Pour les estimations par les méthodes de régression elles sont limitées car le rapport de la variance additive des pères sur leur variance phénotypique est voisin du rapport de la variance additive de l'ensemble de la population sur sa variance phénotypique. En effet, un effort de sélection diminue la variance phénotypique de l'échantillon sélectionné et réduit, pour un caractère héritable, dans des proportions relativement comparables, la variance génétique additive de ce même échantillon. Ce raisonnement intuitif s'est d'ailleurs trouvé en partie confirmé par les expériences de stimulation de RonNingen (I972).

En revanche dans le cas de l'estimation par la composante paternelle de la variance des descendants, la variance additive des pères n'est plus divisée par leur variance phénotypique mais par celle de leurs descendants qui, si les pères sont sélectionnés, lui est de beaucoup supérieure. Dans ce cas il y aura donc généralement une sousestimation importante de l'héritabilité. Cependant, l'introduction, dans nos estimations, du rapport de la variance phénotypique des descendants sur celle des pères, nous a permis de corriger ce biais.

De cette discussion, il apparaît que les valeurs d'héritabilité trouvées, sans doute légèrement surestimées, paraissent néanmoins appréciables. Les valeurs à retenir soit $h^{2}=0,30$ pour le Logarithme naturel du gain et $h^{2}=0,40$ pour le Logarithme naturel du gain moyen par départ, correspondent aux valeurs moyennes et plutôt fortes qui se dégagent de 1'ensemble des données bibliographiques (tab1. 7). Elles confirment que les gains sont de bons estimateurs de l'aptitude à la course plate, 
TABLEAU 7

Héritabilité de la vitesse au galop

\begin{tabular}{|c|c|c|c|c|}
\hline Référence & Pays & Méthode d'estimation & Critère utilisé & $h^{2}$ \\
\hline ARTz (1961) & All. & $\begin{array}{l}\text { Régression sur } 200 \text { couples mère-fille } \\
\text { Analyse de variance sur } 6551 / 2 \text { sœurs } \\
\text { filles de } 31 \text { étalons }\end{array}$ & $\begin{array}{c}\text { temps corrigé } \\
-\end{array}$ & $\begin{array}{l}0,24 \\
0,19\end{array}$ \\
\hline BormanN (1964) & All. & $\begin{array}{l}\text { Analyse de variance } 1 / 2 \text { frères fils de } \\
35 \text { étalons : } \\
\text { en fonction de l'âge : } 2 \text { ans } \\
3 \text { ans } \\
4 \text { ans }\end{array}$ & $\begin{array}{c}\text { temps corrigé } \\
\text { - }\end{array}$ & $\begin{array}{l}\mathbf{0 , 1 7} \\
\mathbf{0 , 0 9} \\
\mathbf{0 , 1 7}\end{array}$ \\
\hline BormanN (1965) & All. & $\begin{array}{l}\text { Analyse de variance sur } 1 / 2 \text { frère } \\
\text { en fonction de l'âge : } 2 \text { ans } \\
3 \text { ans } \\
\text { Régression mère-descendant }\end{array}$ & $\begin{array}{c}\text { temps corrigé } \\
-\end{array}$ & $\begin{array}{l}\mathbf{0 , 0 6} \\
0,14 \\
\mathbf{0 , 0 8}\end{array}$ \\
\hline WATANABE (1968) & Jap. & $\begin{array}{l}\text { Analyse de variance sur } 1 / 2 \text { frère }(500 \\
\text { chevaux de } 4 \text { ans placés de } 1964 \\
\text { à 1966) }\end{array}$ & temps corrigé & 0,12 \\
\hline PERN (1970) & U.R.S.S. & $\begin{array}{l}\text { Diverses méthodes. Régression, corré- } \\
\text { lation, analyse de variance }\end{array}$ & vitesse & $\begin{array}{r}0,05 \\
\text { à } 0,80\end{array}$ \\
\hline Ocsag et Totri (1959) & Hon. & Régression mère-produit & vitesse & $\begin{array}{r}0,04 \\
\text { à } 0,06\end{array}$ \\
\hline $\begin{array}{l}\text { WATANABE et al. }(1966) \\
\text { (cité par DusEK, 1970) }\end{array}$ & Jap. & - & gains & 0,45 \\
\hline PirRi et Stefie (1951) & U.S.A. & $\begin{array}{l}\text { Analyse de variance sur } 23521 / 2 \text { frères } \\
\text { de } 3 \text { ans, fils de } 199 \text { étalons }\end{array}$ & $\begin{array}{l}\text { transformation } \\
\text { logarithmique } \\
\text { des gains }\end{array}$ & 0,60 \\
\hline FoYE et al. (1973) & U.S.A. & D'après la répétabilité des gains & gain moyen/départ & 0,30 \\
\hline BORMANN (1966) & All. & Régression mère-descendant & poids handicap & 0,51 \\
\hline DUSEK (1963) & Tchec. & $\begin{array}{l}\text { Régression mère-fille sur } 125 \text { couples } \\
\text { Régression mère-fille sur } 121 \text { couples }\end{array}$ & handicap général & $\begin{array}{l}0,25 \\
0,45\end{array}$ \\
\hline DUSEK (1965) & Tchec. & $\begin{array}{l}\text { Analyse de variance sur } 148 \quad 1 / 2 \text { frères, } \\
\text { fils de } 8 \text { etalons } \\
\text { Analyse de variance sur } 156 \quad 1 / 2 \text { sœurs } \\
\text { de } 9 \text { étalons }\end{array}$ & handicap général & $\begin{array}{l}0,19 \\
0,25\end{array}$ \\
\hline Foye et al. (1973) & U.S.A. & $\begin{array}{l}\text { Analyse de variance sur } 39 \text { paires de } \\
\text { pleins frères } \\
1 / 2 \text { frères de } 3 \text { ans issus de } 75 \text { étalons } \\
\text { Moyenne pondérée pa! l'auteur }\end{array}$ & $\begin{array}{c}\text { valeur cotée } \\
- \\
-\end{array}$ & $\begin{array}{l}0,36 \\
0,68 \\
0,55\end{array}$ \\
\hline $\begin{array}{l}\text { MORE O'Ferral et } \\
\text { CUNNINGHAM (1974) }\end{array}$ & Irl. & $\begin{array}{l}\text { Régression mère-descendant } \\
\text { Régression parent moyen-descendant } \\
1 / 2 \text { frères paternels } \\
\text { Régression père-descendant }\end{array}$ & $\begin{array}{c}\text { time-form } \\
- \\
-\end{array}$ & $\begin{array}{l}0,36 \\
0,34 \\
0,35 \\
0,56\end{array}$ \\
\hline $\begin{array}{l}\text { SCHWARK et NEISSER } \\
\text { (1971) }\end{array}$ & All. & $\begin{array}{l}\text { Régression mère-fille sur } 153,2 \text { ans } \\
\text { Régression mère-fille sur } 67,3 \text { ans }\end{array}$ & poids handicap & $\begin{array}{l}0,60 \\
0,60\end{array}$ \\
\hline WATANABE (1974) & Jap. & $\begin{array}{l}\text { Analyse de variance sur } 293 \text { produits } \\
\text { de } 48 \text { étalons }\end{array}$ & valeur cotée & 0,64 \\
\hline
\end{tabular}


ce que les rares estimations d'héritabilité disponibles (PIRRI et STEELE, I95I ; WATANABE et al., I966 ; FoyE et al., r973) pouvaient laisser prévoir. Elles sont par ailleurs les premières de la littérature à prendre en considération les croisements assortis qui sont dans bien des cas une source de surestimations. Cependant il est possible qu'une partie de la variance génétique mise ainsi en évidence soit seulement le résultat d'une interaction génotype-milieu. Il n'est pas exclu en effet que les individus au meilleur potentiel bénéficient également des conditions de milieu les plus favorables. Ceci est même probable les soins donnés à ces animaux et leur exploitation étant excessivement " personnalisés ". Néanmoins nous pensons que ces variables, surtout la dernière, autorisent à proposer des classements d'étalons sur leur valeur génétique. En effet, le Logarithme naturel du gain moyen par départ d'un cheval de Pur sang anglais à 3 ans apparaît à la suite de cette étude comme le meilleur critère d'estimation de son potentiel qui soit largement disponible. La prise en compte du nombre de départs augmentant l'héritabilité paraît donc réduire sensiblement les fluctuations des gains dues au nombre de sorties et vraisemblablement aussi à la " pratique du tour " dans les courses à handicap. Par ailleurs, la transformation logarithmique réduisant la variance excessive de la variable gain, hors de proportions avec celle du potentiel des chevaux, permet, elle aussi, d'éliminer une grande partie des fluctuations aléatoires. Cette variable peut donc servir à une indexation génétique généralisée des animaux du Stud-Book. Cependant il faudra tenir compte dans ces estimations de 1'homogamie marquée de la population. Mais nous n'aborderons pas ici ce problème délicat de l'indexation génétique dans le cas d'une population homogame. Ceci fera l'objet d'une étude ultérieure.

Reçu pour publication en décembre 1975.

\section{REMERCIEMENTS}

J'exprime ma gratitude aux responsables de la Société d'encouragement à l'élevage des races de chevaux en France qui ont mis à notre disposition leurs fichiers de recueil des performances.

Je remercie également M. Ch. L.egault de l'encadrement dont il m'a fait bénéficier et M. C. Chevalet dont les critiques constructives ont contribué efficacement à la mise au point définitive de cet article.

\section{SUMMARY}

\section{STATISTICAI, AND GENETICAI, ANALYSIS OF EARNINGS OF THREE-YEARS-OLD THOROUGHBREDS IN FRENCH FLAT RACES FROM I97I TO I973}

Earnings recorded in French flat races of three years old Thoroughbreds from I971 to 1973 as well as those of their parents at the same age have been analysed in order to give different estimates of heritability of this criteria.

Before being submitted to usual statistical analysis, logarithmic transformation has been applied to the earnings of each horse and to its average earning per start. Parent's earnings had been actualized to 1973 by the multiplicative method. 
The analysis of variance of the transformed variables on a sample of 5235 winning progenies allows to determine that the year effect was not significant whereas the sex effect was highly significant in favour of the males. Futhermore, the absence of interaction effects allowed the adjustement of the two variables.

Phenotypic correlation between mates was respectively : .23, .1 $8, .25$ and .35 for earning, average earning per start, Log of earning and Log of average earning per start.

The " stallion " components of variance of mares estimated on 3377 dams and 341 sires were respectively : .I7 for Log of earnings and .26 for Log of average earning per start.

Analysis of variance was then made on the progenies phenotypic values. Three samples selected on a minimum number of winners per sire of respectively 2,4 and 8 were used. These three analyses concerned repectively 5.022, 4.639 and 3.777 offsprings from 507,343 and 220 stallions. "Stallion " components of variance varied from . I 3 to .I 5 for the first variable and from .2 I to .24 for the second one.

Regression coefficients were estimated for sire-offspring (3.84 I pairs), dam-offspring (2.728 pairs) and mid-parent-offspring (2.244 pairs). Correction for assortative matings according to FISHER's method (I9I8) was then applied to the preceeding results. Heritability estimates by all different methods are : less than .ro for untransformed variables, close to .30 fo: Log of earning and close to .40 for Log of average earning per start.

\section{RÉFÉRENCES BIBLIOGRAPHIQUES}

ARtz W., rg6r. Ein Beitrag zur Auswertung der Leistungsfrüfungen in der Vollblutzucht unter besonderer Berücksichtigung der Rennleistungen einzelner Hengstnachkommenschaften. Giessener Schriftenr. Tierz. und Haustiergenet., 2, $62 \mathrm{p}$.

Bormand P., 1964. Die Anwendung biomathematisher Methoden bei der Auswertung der Rennleistung von Vollblutpferden. Ergeb. Landwirtsh. Forsch. Justus-Leibig-Univ., n ${ }^{\circ} 6,76-78$.

Bormans P., I966. Ein Vergleich Zwischen Generalausgleichsgewicht und zeitmessung als Selektionsmassstab in der Vollblutzucht. Züchtungskunde, 38, 301-3ro.

Crow J. F., Kimura M., I970. An introduction to population genetics Theory. Harper et Row, New-York, 575 p.

Dusek J., r963. Bemerkungen zur Beurteilung der Leistungs fähigkeit von Pferden. Schriftenr. MaxPlanck-Inst. Tierz. Tierernaehr., Vol. Spec., 257-284.

Dusek J., 1965. Contribution à l'étude de l'héritabilité de quelques propriétés du Cheval. Zivocisna vyroba, 6, 449-456.

Dusek J., 1965. Estimation des vitesses atteintes par les Pur sang anglais dans les courses plates, d'après les temps obtenus sur l'hippodrome national de Chuchle. Véd. pr. V. S. ch. K., I43-I6o.

Dusek J., I 966 . Analyse de la reproductibilité des performances des chevaux de Pur sang anglais. Véd. pr. I. S. ch. K., Bull., 2, I 26-144.

Dusek J., 1967. Verwendung der Robertsonschen Methode des Jahrgansstallgefährtinnenvergleichs zur Kontrolle der Vererbung in der Pferdezucht. Bayer. Landwirtsch. Jahrb., 44, 875-88o.

DUSEK J., I968. On the question of using the performance of english thoroughbred for the estimation of their breeding value. Pour la science agricole socialiste, 17, 237-257.

Dusek J., r97o. Zur Heritabilität des Körperbaues und des Ganges bei Pferden. Z. Tierz. Zuchtbiol., 87, 14-19.

FALCONER D. S., 1964. Introduction to quantitative genetics. Oliver and Boyd, Edinburgh, London, $365 \mathrm{p}$.

Fisher R. A., 1918. The correlation between relatives on the supposition of Mendelian inheritance. Trans. Roy. Soc. Edinb., 52, 399-443 (cité d'après Crow et KImURA, I970).

Foye D. B., Dickey H. C., Sniffen C. J., I973. Heritability of racing performance and a selection index for breeding potential in the Thoroughbred horse. J. Anim. Sci., 35, II4I-I I45.

Kempthorne O., I957. An introduction to genetic statistics. John Wiley and Sons, New York, 545 p.

Langlois B., 1973. Caractères quantitatifs chez le Cheval : aspects génétiques. Bull. tech. Dép. Genet. anim. (Inst. Nat. Rech. agron., Fr.), $\mathrm{n}^{\circ}$ I6, I35 p.

LANGiors B., I975. Interprétation statistique et génétique des gains des chevaux dans les compétitions équestres françaises. Livest. Prod. Sci., 2, rgr-204.

More O'Ferrall G. J., Cunningham E. P., 1974. Heritability of racing performance in Thoroughbred horses. Livest. Prod. Sci., 1, 87-97.

Ocsag I., Tотн I., r959. The heritability of speed in horses. Agrartud egy. mezögtud Kar Közl., Gödöllö, 61-67. Abstr. in Anim. Breed Abstr., 30, nº 1562.

Pern E. M., I97r. L'héritabilité de la vitesse chez le Pur sang anglais. Genetika, 6, IIo-II4. 
PIRRi J. Jr, Steele, I95I. Heritability of racing capacity in thoroughbreds. J. Anim. Sci., 10, 1029 (Abstr.).

Reeve E. C. R., 1955. Contribution to discussion. Cold. Spr. Harb. Symp. Quant. Biol., $20,76-78$.

ReEve E. C. R., 196r. A note on non-random mating in progeny tests. Genet. Res., Camb., 2, 195-203.

RoNNINGEN K., r972. The effect of selection on heritabilities estimated by twice the parent offspring regressions or twice the parent offspring correlations. Acta Agric. Scand., 22, 200-204.

Watanabe Y., 1969. Zeitmessung als Selektionsmassstab in der Vollblutzucht. Jap. J. Zootech. Sci., 40, $27 \mathrm{I}-276$.

Watanabe Y. et al., 1966 . (cité d'après Dusek, 1970).

Watanabe Y., I974. Performance rates of Thoroughbreds as a criterion of racing ability. Jap. J. Zootech. Sci., 45, 408-4II.

\section{ANNEXE}

Méthode d'estimation de l'héritabilité par la composante paternelle de la variance dans le cas d'une population homogame où les reproducteurs mâles et femelles sont soumis à des pressions de sélection différentes :

Nous savons qu'un individu reçoit en moyenne la moitié de la valeur génétique additive de son père et de sa mère. Nous pouvons donc écrire l'équation de régression suivante :

$$
\mathrm{A}_{d}=\mathrm{I} / 2 \mathrm{~A}_{p}+\mathrm{I} / 2 \mathrm{~A}_{m}
$$

dans laquelle le symbole $\mathrm{A}$ représente les valeurs génétiques additives des individus, les indices $d, p$ et $m$ les affectant respectivement à un descendant, un père et une mère.

Ainsi en considérant deux descendants issus d'un même père, nous pouvons écrire :

$$
\operatorname{Cov}\left(A_{d}, A_{d^{\prime}}\right)=\mathbf{r} / 4 \operatorname{Var}\left(A_{p}\right)+\mathbf{I} / 2 \operatorname{Cov}\left(A_{p}, A_{m}\right)+\mathbf{I} / 4 \operatorname{Cov}\left(A_{m}, A_{m^{\prime}}\right)\left({ }^{1}\right)
$$

les deux descendants et les deux mères considérées étant distingués par un signe prime (').

Par ailleurs si l'on peut supposer l'additivité génétique du caractère et l'absence d'effets de milieu commun sur les descendants d'un même père, nous pourrons écrire :

$$
\operatorname{Cov}\left(\mathrm{A}_{d}, \mathrm{~A}_{d^{\prime}}\right)=\operatorname{Cov}\left(\mathrm{P}_{d}, \mathrm{P}_{d^{\prime}}\right)
$$

où $\mathrm{P}_{d}$ et $\mathrm{P}_{d^{\prime}}$ sont les valeurs phénotypiques des deux descendants considérés.

Si nous posons :

et :

$$
\begin{aligned}
\operatorname{Var}\left(\mathrm{P}_{d}\right) & =\mathrm{R} \operatorname{Var}\left(\mathrm{P}_{p}\right) \\
\operatorname{Var}\left(\mathrm{A}_{m}\right) & =\mathrm{K} \operatorname{Var}\left(\mathrm{A}_{p}\right)
\end{aligned}
$$

et que nous divisons en tenant compte de l'équation (2) les deux membres de l'équation $(\mathrm{I})$ par $\operatorname{Var}\left(\mathrm{P}_{d}\right)$, nous obtenons :

$$
t_{a}=\frac{\mathrm{I}}{4 \mathrm{R}} h^{2}\left(\mathrm{I}+2 r_{\mathrm{A}} \sqrt{\mathrm{K}}+\mathrm{K} t_{\mathrm{A} m}\right)
$$

( $\left.{ }^{1}\right)$ Les symboles $\operatorname{Var}(\mathrm{X})$ et $\operatorname{Cov}(\mathrm{X}, \mathrm{Y})$ représentent respectivement la variance de la variable $\mathrm{X}$ et la covariance des variables $\mathrm{X}$ et $\mathrm{Y}$. 
dans laquelle :

$t_{d}=\operatorname{Cov}\left(\mathrm{P}_{d}, \mathrm{P}_{d^{\prime}}\right) / \operatorname{Var}\left(\mathrm{P}_{d}\right)$ est le coefficient de corrélation intra-classe de père du phénotype des descendants;

$\mathrm{R}=\operatorname{Var}\left(\mathrm{P}_{\boldsymbol{d}}\right) / \operatorname{Var}\left(\mathrm{P}_{\boldsymbol{p}}\right)$ a été défini précédemment ;

$h^{2}$ est l'héritabilité estimée ici par le rapport $\operatorname{Var}\left(\mathrm{A}_{p}\right) / \operatorname{Var}\left(\mathrm{P}_{p}\right)$

$r_{A}$ est la corrélation entre les valeurs génétiques $A_{p}$ et $A_{m}$;

$\mathrm{K}=\operatorname{Var}\left(\mathrm{A}_{m}\right) / \operatorname{Var}\left(\mathrm{A}_{p}\right)$ a également été défini plus haut ;

$t_{\mathrm{A} m}=\operatorname{Cov}\left(\mathrm{A}_{m}, \mathrm{~A}_{m^{\prime}}\right) / \operatorname{Var}\left(\mathrm{A}_{m}\right)$ est la corrélation intra-classe des valeurs génétiques additives des conjointes d'un même père.

Si dans l'équation (3) nous remplaçons $r_{\mathrm{A}}$ et $t_{\mathrm{A} m}$ par leurs estimations $h^{2} r$ et $h^{2} t_{m}$ en fonction des paramètres phénotypiques $r$ et $t_{m}$ correspondants, nous sommes conduits pour estimer l'héritabilité à une équation du second degré en $h^{2}$ soit :

$$
\left(2 r \sqrt{\mathrm{K}}+\mathrm{K} t_{m}\right) h^{4}+h^{2}-4 \mathrm{R} t_{d}=0
$$

dont la racine positive comprise entre $o$ et $\mathrm{I}$ fournit la solution.

En l'absence de sélection, $\mathrm{K}=\mathrm{I}, \mathrm{R}=\mathrm{I}$ et l'équation (4) devient :

$$
\left(2 r+t_{m}\right) h^{4}+h^{2}-4 t_{d}=0
$$

En panmixie et lorsque les descendants considérés sont des demi-frères, $t_{m}=0$ et $r=0$. Nous retrouvons alors le résultat classique $h^{2}=4 t_{d}$.

Dans le cas de pleins frères lorsque $\mathrm{K}=\mathrm{I}, \mathrm{R}=\mathrm{I}$ et $r=0$, la condition $t_{m}=\mathrm{I}$, nécessaire, n'est pas suffisante et il faut remonter à l'équation (3) en faisant $t_{\mathrm{A} m}=\mathrm{I}$ pour retrouver l'estimation classique $h^{2}=2 t_{d}$. 